Radiotherapy electron beams collimated by small tubular applicators: characterization by silicon and diamond diodes

This content has been downloaded from IOPscience. Please scroll down to see the full text. 2013 Phys. Med. Biol. 588121

(http://iopscience.iop.org/0031-9155/58/22/8121)

View the table of contents for this issue, or go to the journal homepage for more

Download details:

IP Address: 192.107.76.5

This content was downloaded on 08/11/2013 at $14: 38$

Please note that terms and conditions apply. 


\title{
Radiotherapy electron beams collimated by small tubular applicators: characterization by silicon and diamond diodes
}

\author{
P Bagalà ${ }^{1}$, C Di Venanzio ${ }^{2}$, M D Falco ${ }^{1}$, A S Guerra ${ }^{3}$, Marco Marinelli ${ }^{2}$, \\ E Milani $^{2}$, M Pimpinella $^{3}$, F Pompili $^{2}$, G Prestopino $^{2}$, R Santoni $^{1}$, \\ $A$ Tonnetti ${ }^{2}, C_{\text {Verona }}{ }^{2}$ and $G$ Verona-Rinati ${ }^{2}$ \\ ${ }^{1}$ Department of Diagnostic Imaging, Molecular Imaging, Interventional Radiology and \\ Radiotherapy, Tor Vergata University General Hospital, Viale Oxford 81, I-00133 Rome, Italy \\ 2 INFN-Dipartimento di Ingegneria Industriale, Università di Roma 'Tor Vergata', \\ Via del Politecnico 1, I-00133 Roma, Italy \\ ${ }^{3}$ Istituto Nazionale di Metrologia delle Radiazioni Ionizzanti, ENEA-INMRI C R Casaccia, \\ Via Anguillarese 301, I-00123 Roma, Italy \\ E-mail: gianluca.verona.rinati@uniroma2.it
}

Received 22 May 2013, in final form 27 September 2013

Published 7 November 2013

Online at stacks.iop.org/PMB/58/8121

\begin{abstract}
High-energy electron beams generated by linear accelerators, typically in the range 6 to $20 \mathrm{MeV}$, are used in small field sizes for radiotherapy of localized superficial tumors. Unshielded silicon diodes (Si-D) are commonly considered suitable detectors for relative dose measurements in small electron fields due to their high spatial resolution. Recently, a novel synthetic single crystal diamond diode (SCDD) showed suitable properties for standard electron beams and small photon beams dosimetry. The aim of the present study is twofold: to characterize 6 to $15 \mathrm{MeV}$ small electron beams shaped by using commercial tubular applicators with 2, 3, 4 and $5 \mathrm{~cm}$ diameter and to assess the dosimetric performance under such irradiation conditions of the novel SCDD dosimeter by comparison with commercially available dosimeters, namely a Si-D and a plane-parallel ionization chamber. Percentage depth dose curves, beam profiles and output factors (OFs) were measured. A good agreement among the dosimeters was observed in all of the performed measurements. As for the tubular applicators, two main effects were evidenced: (i) OFs larger than unity were measured for a number of field sizes and energies, with values up to about 1.3 , that is an output $30 \%$ greater than that obtained at the $10 \times 10 \mathrm{~cm}^{2}$ reference field; (ii) for each diameter of the tubular applicator a noticeable increase of the OF values was observed with increasing beam energy, up to about $100 \%$ in the case of the smaller applicator. This OF behavior is remarkably different from what typically observed for small blocked fields having the same size and energy as those used in this study. OFs for tubular applicators depend considerably on the field size, so interpolation is unadvisable to predict the linear accelerator output for such applicators whereas reliable high-resolution detectors, as the
\end{abstract}


silicon and diamond diodes used in this work allow OF measurements with uncertainties of about $1 \%$.

(Some figures may appear in colour only in the online journal)

\section{Introduction}

In clinical practice, high-energy electron beams generated by linear accelerators (linac) typically in the range 6 to $20 \mathrm{MeV}$ are primarily used for the treatment of superficial cancerous lesions (Vaeth and Meyer 1991, Tapley 1976, Hogstrom and Almond 2006). They are typically shaped to the target region by means of fixed-field-size standard applicators, fastened to the head of the accelerator. In the case of small superficial tumor treatments, tubular small size electron applicators fastened to the linac head can be used. However, a systematic study of small electron beams shaped by such long tubular applicators from 2 to $5 \mathrm{~cm}$ diameter, has not been performed so far.

Plane-parallel ionization chambers are the typical reference dosimeters for high-energy electron beams (IAEA 2000, Thwaites et al 2003, AAPM 1991, 2009), but they are not capable of a sufficiently high lateral spatial resolution due to their large collecting electrode. This is not the case for silicon diodes (Si-D), characterized by small sensitive volume, high sensitivity and high spatial resolution. For these reasons $\mathrm{Si}-\mathrm{D}$ are well assessed in clinical relative electron dosimetry (Griessbach et al 2005, Evelin et al 1999, Rikner 1985). However, it is worth noting that a dependence of their response on beam energy, dose rate and radiation incidence angle has been reported in literature for some types of Si-D (Grusell and Rikner 1993, Björk et al 2000, Saini and Zhu 2004). Such a dependence can lead to incorrect dose evaluations in depth-dose and beam profiles measurements if appropriate correction factors are not applied (Song et al 2006).

The synthetic single crystal diamond diode (SCDD) developed in the laboratories of the University of Rome 'Tor Vergata', may be an appropriate detector for measurements in high-energy small-field electron beams (Almaviva et al 2010). The suitability of this smallvolume detector for dosimetry in standard clinical beams was already investigated, showing very good dosimetric properties in comparison with reference detectors (Almaviva et al 2008, Di Venanzio et al 2013). In addition, such SCDDs were characterized in small photon beams (down to $1 \times 1 \mathrm{~cm}^{2}$ ), whose dosimetry is challenging (Ciancaglioni et al 2012, Pimpinella et al 2012a, 2012b). The obtained results showed that, due to its high spatial resolution, SCDD is an adequate candidate for dosimetry of small photon fields as well.

The aim of this work is the dosimetric characterization of clinical electron beams from 6 to $15 \mathrm{MeV}$, generated by a linear accelerator, in small field size from 2 to $5 \mathrm{~cm}$ diameter, shaped by means of commercial tubular applicators. Such a characterization was accomplished by means of an unshielded commercial silicon diode and a novel synthetic SCDD dosimeter and the measurement results of the two detectors were compared each other.

\section{Materials and methods}

\subsection{Linear accelerator and tubular applicators}

High-energy electron beams were generated by an Elekta Precise linear accelerator (Elekta Crawley, UK) at 'Tor Vergata' General Hospital in Rome. Dose measurements were carried out for $6,8,10,12$ and $15 \mathrm{MeV}$ electron beams by using both the reference $10 \times 10 \mathrm{~cm}^{2}$ field and 


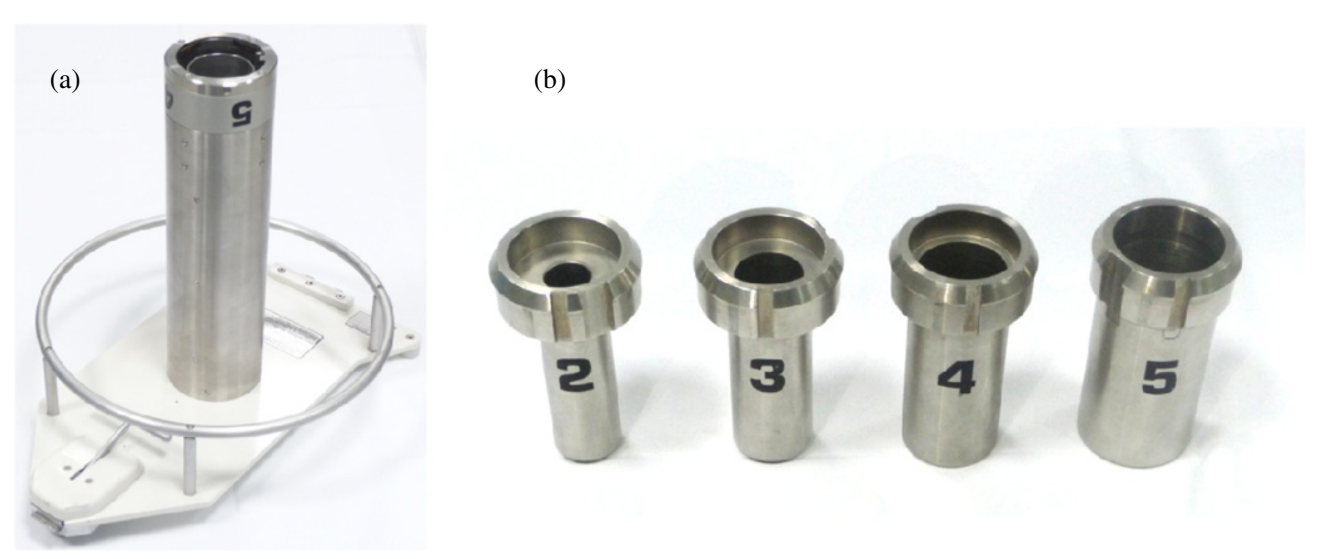

Figure 1. Tubular applicator to be fastened to the accelerator (a) and end tubes with diameter ranging from 2 to $5 \mathrm{~cm}(\mathrm{~b})$.

small-size circular fields. The former was obtained by using the standard $10 \times 10 \mathrm{~cm}^{2}$ square applicator fastened to the head of the accelerator. Small-size circular fields were instead obtained by a fixed x-ray collimator (i.e. upper jaws) setting, a main tubular electron applicator fastened to the head of the accelerator and a set of add-on field defining end tubes with diameter of 2, 3, 4 and $5 \mathrm{~cm}$, inserted at the end of the main tubular applicator (Elekta Crawley, UK). The main applicator has an internal diameter of about $66 \mathrm{~mm}$ and is $337 \mathrm{~mm}$ long with no end tube inserted, reaching an overall length of $438 \mathrm{~mm}$ with the end tube in position. Both the main applicator and end tubes are made of stainless steel. In figure 1 the main tubular electron applicator (a) and the set of end tubes (b) are displayed.

\subsection{Detectors}

Measurements were performed by a synthetic SCDD and two types of commercial dosimeters: an unshielded $p$-type silicon detector (PTW Diode E type 60017, PTW Freiburg) and a planeparallel ionization chamber (PPC05, Scanditronix Wellhöfer, Germany).

The SCDD is based on a diamond Shottky diode, fabricated at Rome 'Tor Vergata' University laboratories and embedded in the same waterproof housing developed by PTWFreiburg for the unshielded silicon diode detector 60017 (figure 2). The diamond plate consists of a multi-layered highly conductive p-type-diamond/intrinsic-diamond structure fabricated by a two-step microwave plasma enhanced chemical vapor deposition (CVD) process on a commercial $3.0 \times 3.0 \times 0.3 \mathrm{~mm}^{3}$ high-pressure, high-temperature (HPHT) Ib singlecrystal diamond substrate (Di Venanzio et al 2013). The detector sensitive volume is of about $3.8 \times 10^{-3} \mathrm{~mm}^{3}$. A thorough study of the physical properties and detection mechanism of such a device is reported elsewhere (Almaviva et al 2010). In all of the measurements reported in the present paper the SCDD was operated in photovoltaic mode, i.e. with no external bias voltage applied. The reference measurement point was assumed at the center of the top surface of the intrinsic diamond layer, $1.3 \mathrm{~mm}$ below the detector tip. The diamond detector was positioned with its main axis parallel to the beam central axis (vertical orientation) to minimize possible stem effects.

The PTW diode E ( $\mathrm{Si}-\mathrm{D})$ is a solid state detector and it is considered a suitable dosimeter for dose measurements in small electron fields due to its small size, allowing accurate beam profile and percentage depth dose (PDD) measurements. Its disk-shaped sensitive volume is 
(a)
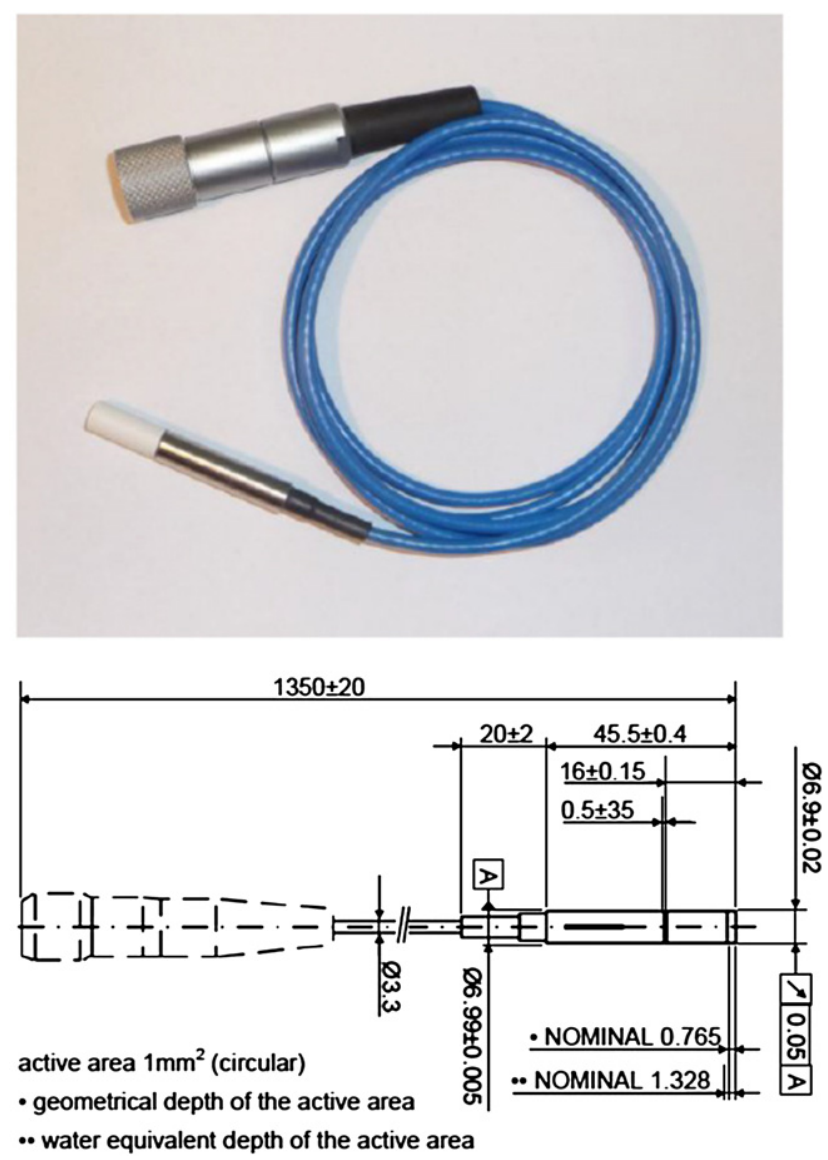

Figure 2. (a) Photo of the SCDD as embedded in the same waterproof housing used by PTWFreiburg for the unshielded silicon diode detector type 60017. (b) Schematic of the PTW 60017 detector.

$0.03 \mathrm{~mm}^{3}$ and the reference point is located on its main axis, $0.77 \mathrm{~mm}$ below the detector tip, which corresponds to an equivalent depth in water of $0.13 \mathrm{~g} \mathrm{~cm}^{-2}$. The silicon detector was used in vertical orientation with no bias voltage applied according to the manufacturer's recommendations.

Both SCDD and Si-D detectors were used for beam profile, depth dose and output factor (OF) measurements in the small circular beams.

The PPC05 is a well guarded plane-parallel ionization chamber for electron dosimetry. It has a $0.05 \mathrm{~cm}^{3}$ nominal volume, $0.6 \mathrm{~mm}$ air cavity height, a $9.9 \mathrm{~mm}$ diameter collecting electrode and a $3.4 \mathrm{~mm}$ guard ring. The reference point of the chamber was assumed at the center of the inside surface of the entrance window (i.e. $1 \mathrm{~mm}$ below the upper surface). During measurements in water phantom the water equivalent thickness of the chamber front wall $\left(0.18 \mathrm{~g} \mathrm{~cm}^{-2}\right)$ was taken into account in establishing the measurement depth in water. According to the manufacturer's recommendations a polarizing voltage of $+300 \mathrm{~V}$ was applied. Plane-parallel air filled ionization chambers, are recommended by dosimetry protocols for high-energy electron beam dosimetry, provided that the field is uniform over the ionization chamber collecting area. In small fields, when performing dosimetric measurements with 
chambers which are large in relation to the field size, volume averaging by the detector has a large influence on the measurement, leading to an underestimation of the measured dose. In the present work, the PPC05 plane-parallel chamber was used as reference dosimeter for OF determination and central-axis depth dose measurements in the $5 \mathrm{~cm}$ diameter circular field and in the $10 \times 10 \mathrm{~cm}^{2}$ standard field, respectively.

\subsection{Dosimetric measurements}

Measurements were performed in a PTW MP3 motorized water phantom at a SSD of $100 \mathrm{~cm}$. The detectors were connected to a PTW TANDEM electrometer and the PTW Mephysto ${ }^{\circledR}$ $\mathrm{MC}^{2}$ software was used both for data acquisition and analysis of beam profiles and depth dose distributions. A PTW Unidos E Universal Dosimeter was used for OF measurements.

PDD curves were measured along the beam central axis both with the SCDD and the Si-D, for 6, 8, 10, 12 and $15 \mathrm{MeV}$ electron beams in 2, 3, 4 and $5 \mathrm{~cm}$ diameter circular field sizes and in the reference field of $10 \times 10 \mathrm{~cm}^{2}$. In the case of the $10 \times 10 \mathrm{~cm}^{2}$ square field, PDDs were also measured by the PPC05 plane-parallel ionization chamber. The alignment of each detector with respect to the central axis was carefully verified by performing both in-plane (i.e. in the gun-target direction) and cross-plane (i.e. perpendicular to the gun-target direction) profile measurements for all the investigated field sizes.

Moreover, since the water-to-carbon and water-to-silicon mass collision stopping power ratios are nearly constant in the range 1-20 MeV, no correction was applied to the PDD curves neither for the SCDD nor for the Si-D (IAEA 2000). Depth ionization curves measured with PPC05 were converted to depth dose curves according to the IAEA TRS-398 protocol (IAEA 2000). The variation of ion recombination and polarity effects with depth was also considered. The main parameters obtained from the PDD curves were $R_{100}$ and $R_{50}$, i.e. the depths in water of the corresponding maximum value of absorbed dose and of the half of its maximum value, the practical range, $R_{p}$, the percentage surface dose, $D_{s}$, and the PDD due to bremsstrahlung, $D_{x}$.

In-plane and cross-plane beam profiles were measured by SCDD and Si-D for each circular field for 6, 8, 10, 12 and $15 \mathrm{MeV}$ electron beams. Each profile was acquired at the depth of maximum dose, $R_{100}$, as derived from PDD measurements in the same irradiation condition. The profiles measured by the two different detectors were compared in terms of $80 \%-20 \%$ penumbra and field size measured at $50 \%$ of the central axis dose value.

OFs were measured by SCDD and Si-D, in 6, 8, 10, 12 and $15 \mathrm{MeV}$ electron beams for all circular fields (2, 3, 4 and $5 \mathrm{~cm}$ diameter). Only in the case of the $5 \mathrm{~cm}$ diameter field size, the PPC05 ionization chamber was also used. The detectors were placed at the depth $R_{100}$ previously determined for each field size. OFs were obtained as the ratio of the dose at $R_{100}$ for the field of interest to the dose at $R_{100}$ for $10 \times 10 \mathrm{~cm}^{2}$ reference field, for the same number of monitor units. For the PPC05 chamber measurements, the variation of water-to-air stopping-power ratio with depth was accounted for in the OF determination (IAEA 2000, Thwaites et al 2003).

\section{Results}

\subsection{Percentage depth dose curves}

Figure 3 shows the PDD curves measured in the reference $10 \times 10 \mathrm{~cm}^{2}$ field using all the dosimeters (Si-D, SCDD and PPC05). The difference in \% between PDDs measured by the diode dosimeters and the ionization chamber are also shown in figure 3. An agreement within 


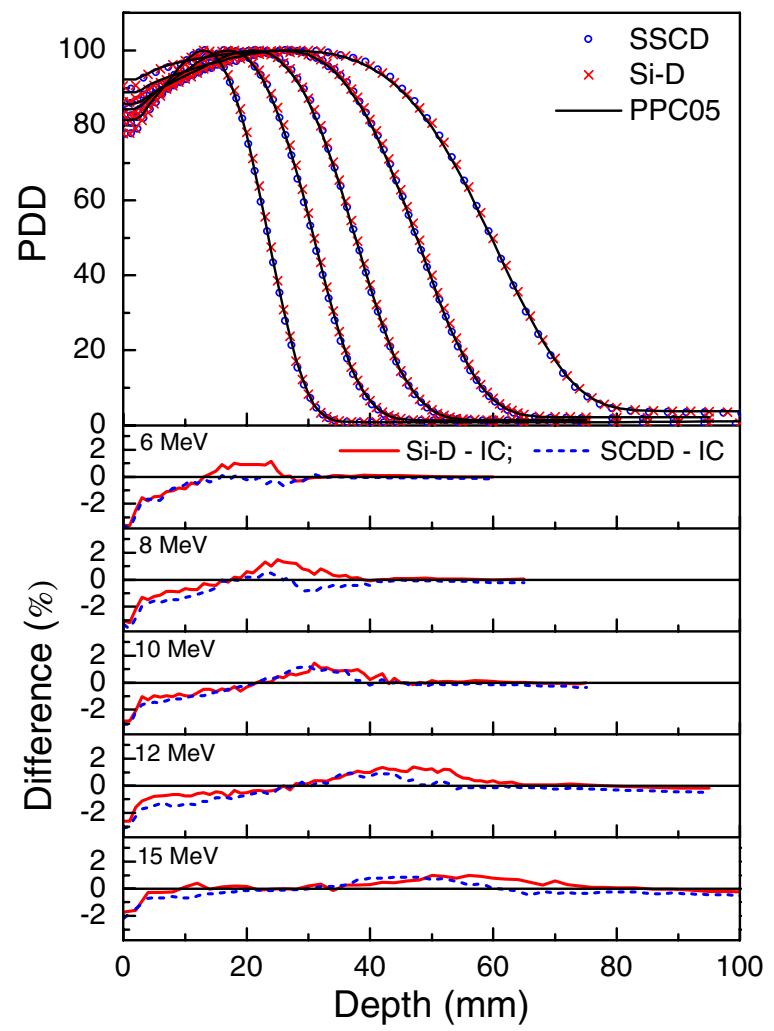

Figure 3. PDD curves measured by Si-D, SCDD diodes and PPC05 plane-parallel ionization chamber for $6,8,10,12$ and $15 \mathrm{MeV}$ electron beams with $10 \times 10 \mathrm{~cm}^{2}$ field size. Difference plots in $\%$ between the diodes and PPC05 ionization chamber are also shown.

$\pm 1.5 \%$ is observed among the detector response for all the beam energies, over the whole PDD curve with the exception of the region in close proximity to the water surface where the detectors are partially in air. In figure 4 the PDD curves measured by Si-D and SCDD in $2 \mathrm{~cm}$ (figure 4(a)) and $5 \mathrm{~cm}$ (figure 4(b)) diameter fields are reported for all energies, together with the respective percentage differences between PDDs measured by the two dosimeters.

PDD curves measured by SCDD and Si-D are reported as a function of field size for 6 and $15 \mathrm{MeV}$ beams in figures 5(a) and (b) respectively. The most relevant dosimetric parameters extracted from PDD curves are summarized in table 1 , showing very similar results by Si-D and SCDD dosimeters.

\subsection{Beam profiles}

Figures 6(a) and (b) show the 6 and $15 \mathrm{MeV}$ in-plane normalized profiles measured by Si-D and SCDD at the $R_{100}$ depth derived for each detector from the PDDs acquired in the same irradiation conditions $\left(R_{100}\right.$ value in table 1$)$. In both cases, profiles were measured for all the available end tubes. The field size and the $80 \%-20 \%$ penumbra value obtained by averaging the left and right hand side values are reported in table 2 for all the profiles of figure 6. 

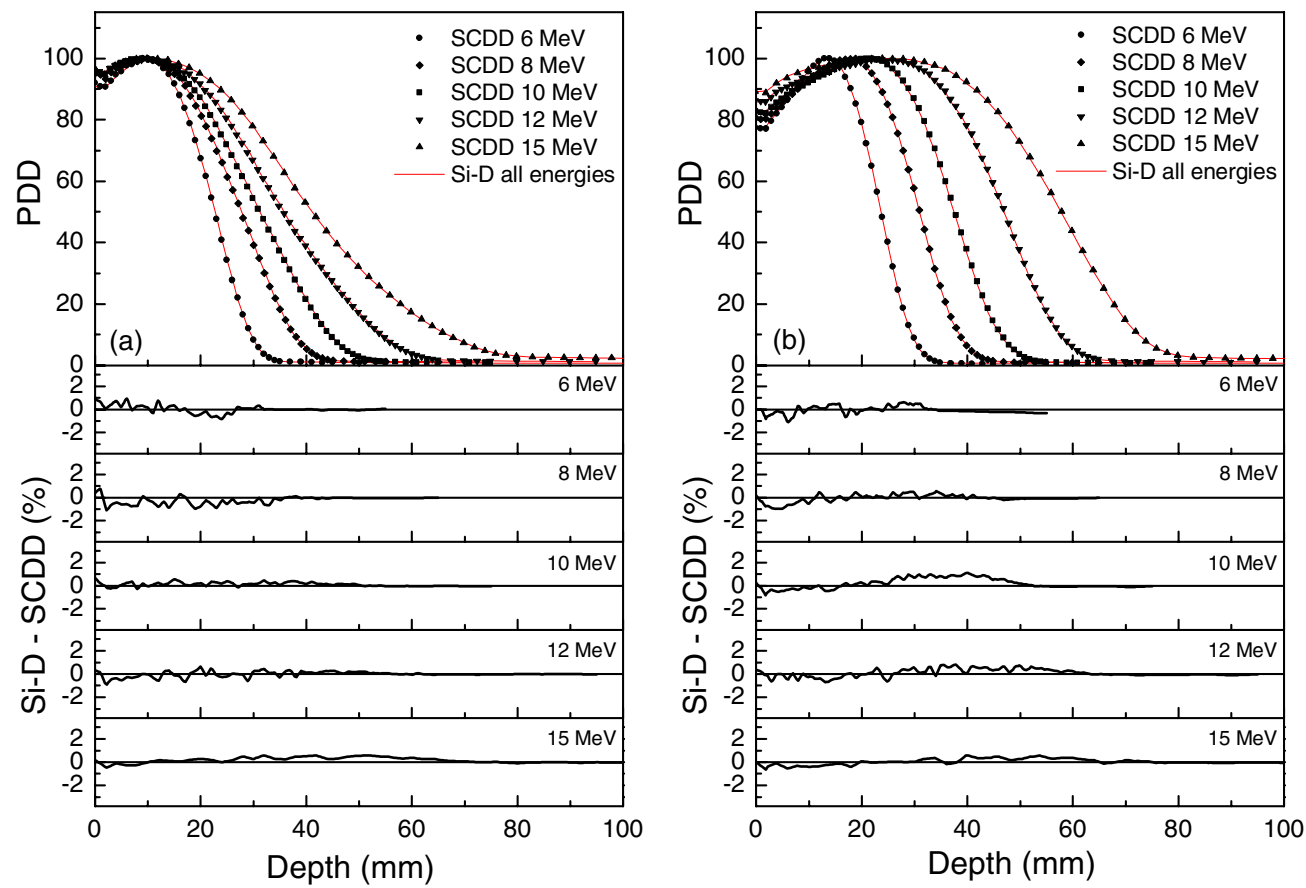

Figure 4. PDD curves for all energies, 2 and $5 \mathrm{~cm}$ diameter field sizes for Si-D and SCDD detectors ( $\mathrm{a}$ and $\mathrm{b}$ ) and their respective difference plots.
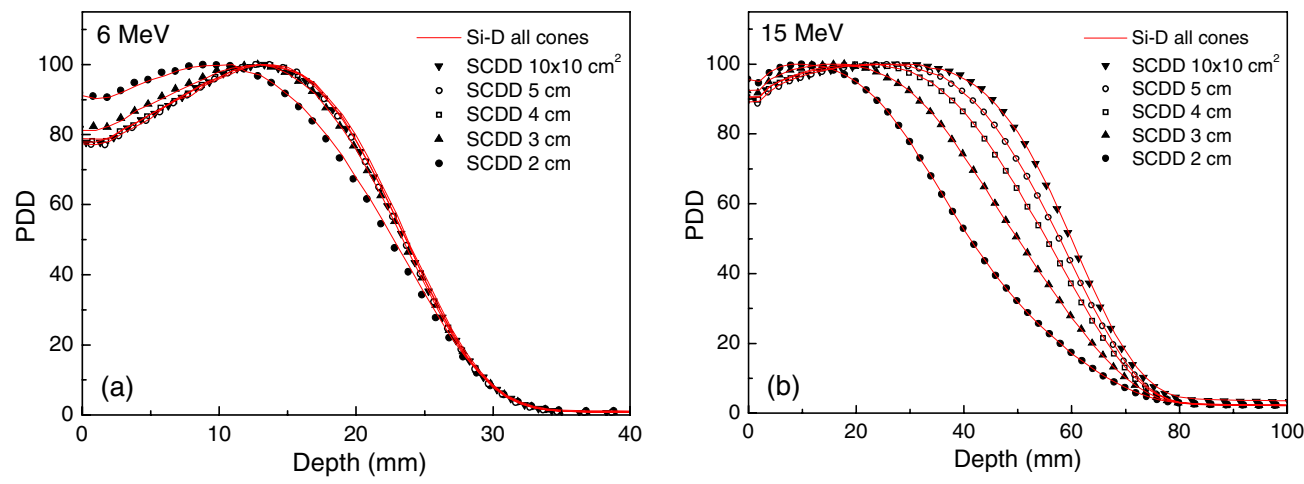

Figure 5. PDD curves measured by the Si-D and the SCDD in all field size diameters investigated for $6 \mathrm{MeV}$ (a) and $15 \mathrm{MeV}$ (b) electron beams. The curves for the $10 \times 10 \mathrm{~cm}^{2}$ field size are also shown.

\subsection{Output factors}

The OF values obtained using all the dosimeters (Si-D, SCDD and PPC05) for all the investigated tubular applicators and beam energies are reported in table 3 . The OF values obtained by Si-D and SCDD are also shown in figure 7, where OF variation with energy and field size is clearly visible.

The reproducibility of the $\mathrm{OF}$ values, obtained in different measurement sessions, resulted $0.5 \%$ ( $1 \mathrm{SD}$ ) for the Si-D and SCDD dosimeters and $0.2 \%$ (1 SD) for the ionization chamber. The uncertainty of the OF values obtained by the ionization chamber was estimated $0.5 \%$ 

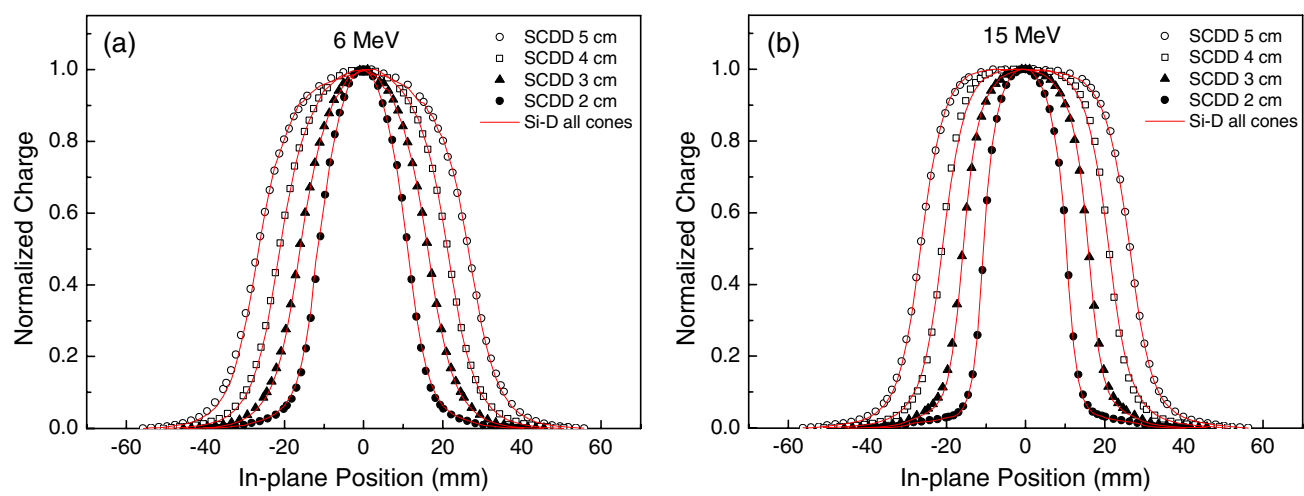

Figure 6. In-plane normalized profiles measured by Si-D and SCDD for $6 \mathrm{MeV}$ (a) and $15 \mathrm{MeV}$ (b) electron beams for all the field size diameters.

Table 1. PDD-extracted parameters for the depth dose curves reported in figure 5 for the different field diameters and the $10 \times 10 \mathrm{~cm}^{2}$ reference field size.

\begin{tabular}{|c|c|c|c|c|c|c|c|c|c|c|}
\hline \multicolumn{11}{|c|}{$6 \mathrm{MeV}$} \\
\hline \multirow[t]{2}{*}{$\underline{\text { Field size }(\mathrm{cm})}$} & \multicolumn{2}{|c|}{2} & \multicolumn{2}{|c|}{3} & \multicolumn{2}{|c|}{4} & \multicolumn{2}{|c|}{5} & \multicolumn{2}{|c|}{$10 \times 10$} \\
\hline & Si-D & SCDD & Si-D & SCDD & Si-D & SCDD & Si-D & SCDD & Si-D & SCDD \\
\hline$R_{100}(\mathrm{~mm})$ & 11.0 & 9.0 & 13.0 & 13.0 & 13.0 & 13.0 & 13.0 & 14.0 & 13.0 & 13.3 \\
\hline$R_{50}(\mathrm{~mm})$ & 22.8 & 22.7 & 23.4 & 23.6 & 23.8 & 23.8 & 23.7 & 23.8 & 23.7 & 23.5 \\
\hline$R_{p}(\mathrm{~mm})$ & 29.6 & 29.7 & 29.5 & 29.7 & 29.6 & 29.9 & 29.5 & 29.8 & 29.5 & 29.6 \\
\hline$D_{s}(\%)$ & 90.7 & 91.5 & 81.2 & 82.5 & 78.8 & 78.3 & 77.2 & 77.3 & 77.9 & 77.8 \\
\hline$\underline{D_{x}}(\%)$ & 1.1 & 1.1 & 0.8 & 0.8 & 0.8 & 0.7 & 0.7 & 0.7 & 0.9 & 0.9 \\
\hline \multicolumn{11}{|c|}{$15 \mathrm{MeV}$} \\
\hline \multirow[t]{2}{*}{ Field size $(\mathrm{cm})$} & \multicolumn{2}{|c|}{2} & \multicolumn{2}{|c|}{3} & \multicolumn{2}{|c|}{4} & \multicolumn{2}{|c|}{5} & \multicolumn{2}{|c|}{$10 \times 10$} \\
\hline & SCDD & Si-D & SCDD & Si-D & SCDD & SCDD & Si-D & SCDD & Si-D & SCDD \\
\hline$R_{100}(\mathrm{~mm})$ & 10.0 & 10.1 & 15.9 & 16.0 & 21.4 & 20.0 & 24.3 & 24.2 & 27.8 & 27.2 \\
\hline$R_{50}(\mathrm{~mm})$ & 41.1 & 41.3 & 50.0 & 50.3 & 55.3 & 55.4 & 57.8 & 58.0 & 60.0 & 59.8 \\
\hline$R_{p}(\mathrm{~mm})$ & 61.6 & 61.8 & 70.1 & 70.5 & 72.6 & 72.8 & 73.0 & 73.1 & 73.0 & 72.0 \\
\hline$D_{s}(\%)$ & 95.5 & 95.4 & 92.5 & 92.5 & 90.6 & 90.4 & 89.2 & 89.1 & 90.6 & 90.1 \\
\hline$D_{x}(\%)$ & 2.9 & 2.9 & 2.6 & 2.6 & 2.7 & 2.6 & 2.7 & 2.6 & 4.1 & 3.9 \\
\hline
\end{tabular}

Table 2. Beam profile parameters from figure 6 .

\begin{tabular}{|c|c|c|c|c|c|c|c|c|}
\hline \multicolumn{9}{|c|}{$6 \mathrm{MeV}$} \\
\hline \multirow[t]{2}{*}{ End tube diameter $(\mathrm{cm})$} & \multicolumn{2}{|c|}{2} & \multicolumn{2}{|c|}{3} & \multicolumn{2}{|c|}{4} & \multicolumn{2}{|c|}{5} \\
\hline & Si-D & SCDD & Si-D & SCDD & Si-D & SCDD & $\mathrm{Si}-\mathrm{D}$ & SCDD \\
\hline Field size $(\mathrm{cm})$ & 2.3 & 2.3 & 3.2 & 3.3 & 4.2 & 4.3 & 5.3 & 5.3 \\
\hline Penumbra (mm) & 8.0 & 7.6 & 10.0 & 10.1 & 10.7 & 10.9 & 11.6 & 12.0 \\
\hline \multicolumn{9}{|c|}{$15 \mathrm{MeV}$} \\
\hline \multirow[t]{2}{*}{ End tube diameter $(\mathrm{cm})$} & \multicolumn{2}{|c|}{2} & \multicolumn{2}{|c|}{3} & \multicolumn{2}{|c|}{4} & \multicolumn{2}{|c|}{5} \\
\hline & Si-D & SCDD & Si-D & SCDD & Si-D & SCDD & Si-D & SCDD \\
\hline Field size (cm) & 2.1 & 2.1 & 3.2 & 3.2 & 4.2 & 4.2 & 5.3 & 5.3 \\
\hline Penumbra (mm) & 4.8 & 4.9 & 6.0 & $6.1^{*}$ & 8.3 & 7.2 & 8.8 & 8.9 \\
\hline
\end{tabular}




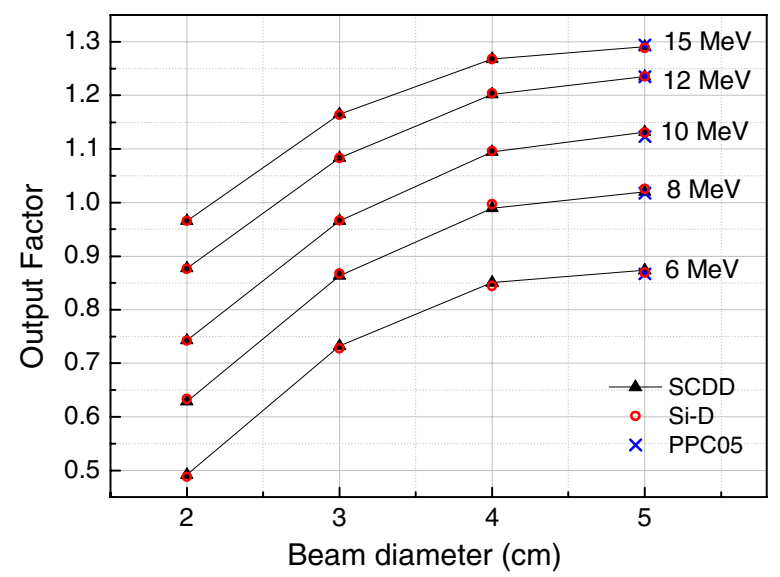

Figure 7. Relative OFs versus field size diameter at $R_{100}$ for each energy and field size, normalized to the $10 \times 10 \mathrm{~cm}^{2}$ field. Measurements were performed using the SCDD, the Si-D and, only for the $5 \mathrm{~cm}$ diameter field size, the PPC05 plane-parallel chamber.

Table 3. OF values for all the tubular applicators normalized to the $10 \times 10 \mathrm{~cm}^{2}$ reference field, measured with Si-D and SCDD for all energies. In the case of $5 \mathrm{~cm}$ applicator, OFs obtained by PPC05 are also reported.

\begin{tabular}{lllllll}
\hline \multirow{2}{*}{ Beam diameter } & & \multicolumn{5}{c}{ Beam energy } \\
\cline { 3 - 7 } & Dosimeter type & $6 \mathrm{MeV}$ & $8 \mathrm{MeV}$ & $10 \mathrm{MeV}$ & $12 \mathrm{MeV}$ & $15 \mathrm{MeV}$ \\
\hline \multirow{2}{*}{$5 \mathrm{~cm}$} & PPC05 & 0.866 & 1.018 & 1.123 & 1.231 & 1.290 \\
& Si-D & 0.869 & 1.025 & 1.130 & 1.235 & 1.288 \\
& SCDD & 0.873 & 1.020 & 1.132 & 1.235 & 1.291 \\
$4 \mathrm{~cm}$ & Si-D & 0.844 & 0.997 & 1.096 & 1.204 & 1.268 \\
$3 \mathrm{~cm}$ & SCDD & 0.851 & 0.989 & 1.095 & 1.202 & 1.268 \\
& Si-D & 0.728 & 0.867 & 0.966 & 1.083 & 1.164 \\
$2 \mathrm{~cm}$ & SCDD & 0.732 & 0.864 & 0.966 & 1.084 & 1.165 \\
& Si-D & 0.488 & 0.633 & 0.742 & 0.876 & 0.966 \\
& SCDD & 0.492 & 0.629 & 0.743 & 0.877 & 0.966 \\
\hline
\end{tabular}

(1 SD) including the uncertainty component associated to the ratio of $s_{w \text {,air }}$ values at different measurement depths (IAEA 2000, Rogers 2004).

\section{Discussion}

A preliminary comparison among the solid state dosimeters and the plane parallel PPC05 ionization chamber was initially performed in order to validate the dosimetric properties of Si-D and SCDD diodes. PDDs curves in the standard $10 \times 10 \mathrm{~cm}^{2}$ field and OFs in the $5 \mathrm{~cm}$ diameter circular beam were measured in the energy range from 6 to $15 \mathrm{MeV}$. As shown in figure 3 , by excluding the region in close proximity to the water surface where the detectors are partially in air, an agreement within $\pm 1.5 \%$ was found among all the detector responses. OFs measured by the solid state detectors agree with those measured with the PPC05 ionization chamber within $0.8 \%$ (see table 3 ). On the basis of these results, the response of both the Si-D and SCDD detectors has been assumed to be energy independent and insensitive to angular distribution of the in-phantom scattered electrons in the energy range considered in this work, with an overall uncertainty of the detector response of about $1.5 \%$. 
The SCDD and the Si-D are characterized by a very small sensitive volume $(0.0038$ and $0.03 \mathrm{~mm}^{3}$ respectively) indicating their suitability for small electron beam dosimetry. This is confirmed by the close agreement found between PDDs measured by the detectors in electron beams with 2 to $5 \mathrm{~cm}$ field diameter. A maximum deviation within about $\pm 1 \%$ can be seen in the difference plots, shown in figure 4 , for all the investigated electron beam energies and field sizes. A good agreement was also observed between the two dosimeters in term of spatial resolution as shown in figure 6 , where the beam profiles are reported for all the field sizes in the case of $6 \mathrm{MeV}$ (figure 6(a)) and $15 \mathrm{MeV}$ (figure 6(b)) beam energy. In this respect, a more quantitative analysis of the obtained results is reported in table 2 . The sizes of field produced by each end tube, derived from profiles measured by the Si-D and the SCDD, agree each other within $0.1 \mathrm{~mm}$. Similarly, a very good agreement $(0.2 \mathrm{~mm})$ is observed between the penumbra values, except for the values referred to three irradiation conditions: $6 \mathrm{MeV}$ with 2 and $5 \mathrm{~cm}$ fields, and $15 \mathrm{MeV}$ with $4 \mathrm{~cm}$ field size where the differences are 0.4, 0.4 and $1.1 \mathrm{~mm}$, respectively. It should be pointed out that, in such conditions, beam profiles were measured at slightly different depths in water when using Si-D and SCDD. As reported in table 1 , in the three above mentioned irradiation conditions the $R_{100}$ values determined by the PDDs measured by the two solid state dosimeters differ up to $2 \mathrm{~mm}$, being all the other values in agreement within $0.1 \mathrm{~mm}$. Such differences in the $R_{100}$ values originate from a small dispersion of the measured data. In the flat region of the PDD curve this dispersion can give rise to a different evaluation of $R_{100}$ even though the curves are very similar. On the other hand, since the scattered radiation at the beam edges increases with water depth, penumbra values are very sensitive to the measurement depth, and this justifies the slightly larger penumbra measured by the detector placed at deeper $R_{100}$.

PDD curves for circular beams shaped by the tubular applicators show a shift of $R_{100}$ toward the surface and a gradient reduction in the fall-off region when the field size decreases as can be seen in figures 5 for the 6 and $15 \mathrm{MeV}$ beams. Such a trend can be explained in terms of the lack of lateral scatter equilibrium (LSE) occurring in small fields, which is known to affect the PDD shape. In particular, when the field size increases, both $R_{100}$ and $R_{50}$ initially increase but become constant beyond the field size corresponding to the achievement of LSE. The minimum field radius for the achievement of LSE at the beam central axis is approximately given by:

$$
R_{\mathrm{eq}} \cong 0.88 \sqrt{E_{p, 0}}
$$

where $R_{\text {eq }}$ is the field radius in $\mathrm{cm}$ and $E_{p, 0}$ is the most probable energy of electron beam at the phantom surface in MeV (Khan and Higgins 2001). $R_{\text {eq }}$ values were determined for all the beam energies considered in this work by using for $E_{p, 0}$ the expression $E_{p, 0}=0.22+1.98$ $R_{p}+0.0025 R_{p}{ }^{2}$ (ICRU 1984). $R_{\text {eq }}$ values are 2.2 and $3.4 \mathrm{~cm}$ for the 6 and $15 \mathrm{MeV}$ beams respectively, corresponding to beam diameters of about 4.4 and $6.8 \mathrm{~cm}$. This is consistent with the results reported in figure 5(a), where no significant differences are observed among PDD curves measured at $6 \mathrm{MeV}$ down to a beam diameter of $4 \mathrm{~cm}$ thus confirming that LSE of the $6 \mathrm{MeV}$ electron beam is reached with tubular applicators of diameter $4 \mathrm{~cm}$. On the other hand, figure 5(b) clearly shows that for the $15 \mathrm{MeV}$ beam all the PDD curves measured in the circular beams differ from the reference curve (i.e. PDD measured in the $10 \times 10 \mathrm{~cm}^{2}$ field size) indicating that LSE is still not achieved in the $5 \mathrm{~cm}$ diameter field size.

As shown in figure 7 and table 3 OF values measured by SCDD and Si-D well agree each other. Differences are typically within $\pm 0.5 \%$ with a maximum value of $0.8 \%$ still consistent with the measurement reproducibility $(0.5 \%, 1 \mathrm{SD})$. This confirms the reliability of the two solid state detectors also in such a kind of measurements. 
It is worth to point out that OFs for tubular applicators resulted to be in some cases larger than unity showing an increase of the output up to $30 \%$ for $5 \mathrm{~cm}$ diameter field size at $15 \mathrm{MeV}$, in comparison with the output of the $10 \times 10 \mathrm{~cm}^{2}$ field size. In addition, a clear increase of the OF values is observed for each diameter of the tubular applicator by increasing beam energy while at fixed energy a decrease of OF can be noticed when reducing the field size.

The observed trend of the OF values can be explained in terms of three competing mechanisms: (i) beam scattering in the water phantom, (ii) scattering of electrons in air and (iii) scattering of electrons into the useful beam by the collimator walls.

Mechanism (i) is known to increase the dose at $R_{100}$ with the field size up to the beam radius for which the LSE condition is reached (Khan 2003, Zhang et al 1999).

When high-energy electrons travel in air, the electron beam is broadened (mechanism ii) due to significant air scattering which is more effective at low energies (mass scattering power $3.38 \times 10^{-2} \operatorname{radian}^{2} \mathrm{~cm}^{2} \mathrm{~g}^{-1}$ at $15 \mathrm{MeV}$ and $1.70 \times 10^{-1} \operatorname{radian}^{2} \mathrm{~cm}^{2} \mathrm{~g}^{-1}$ at $\left.6 \mathrm{MeV}\right)(\mathrm{Lax}$ and Brahme 1980, ICRU 1984). Due to this effect, since tubular applicators of different size are used with a fixed upper jaws setting, the number of air-scattered electrons reaching the end of the collimator at the beam central axis decreases with beam size. As electrons are scattered at larger angles at low energies, for a given field size, this effect tends to lower the OF as the beam energy decreases.

In the case of tubular applicators fastened to the linac head, electrons impinging the collimator walls can be scattered forward in the useful beam (mechanism iii) traveling up to the end of the tubular collimator. This leads to an increase of the electron fluence in comparison with the $10 \times 10 \mathrm{~cm}^{2}$ standard field size. This scattering mechanism is more effective at higher energies since the mean electron scattering angle is lower due to the lower mass scattering power of collimator walls (for ${ }_{26} \mathrm{Fe}$ is $1.05 \mathrm{E}-1 \operatorname{radian}^{2} \mathrm{~cm}^{2} \mathrm{~g}^{-1}$ at $15 \mathrm{MeV}$ and 5.22E-1 radian $\mathrm{cm}^{2} \mathrm{~g}^{-1}$ at $6 \mathrm{MeV}$ ). As a consequence a larger number of electrons are scattered towards the center of the field. The large number of wall-scattered electrons can compensate the decrease of the electron fluence caused by the reduction of field size below the standard field. Then OF values larger than unity can be observed especially at higher energies and for larger end tubes which is indeed the case for the data shown in figure 7.

OFs larger than unity have been reported in literature for long tubular applicators made of low Z materials (i.e. PMMA) used in intraoperative radiotherapy (IORT) (Björk et al 2004, Pimpinella et al 2007). Results are not fully comparable due to the dissimilar geometries and materials of applicators, however it is worth to mention that a large component of collimator-scattered electrons in the useful beam has been reported for those collimators as well as results of Monte Carlo simulations. On the contrary, the phenomenon of OFs larger than unity generally does not take place for similar electron energies and analogous small-size fields obtained by blocking the open fields using lead or Cerrobend cut-outs placed at the end of standard applicators (blocked fields), minimizing the wall-scattered electron component (Rashid et al 1990, Björk et al 2004, Amin et al 2011). In such cases OFs monotonically decrease below unity for field size below $10 \times 10 \mathrm{~cm}^{2}$. On the other hand, due to the different components determining the linac output when tubular applicators are used, the accelerator output depends considerably on field size and energy. Therefore, simple interpolation is inadvisable to predict the linac output when using such applicators and dosimetric measurements should be performed for each condition foreseen for patient irradiation using reliable high-resolution detectors.

It should be noted that, even though the tubular applicators are made of stainless steel, no significant increase of the PDD bremsstrahlung tail was observed in comparison with the standard $10 \times 10 \mathrm{~cm}^{2}$ applicator as shown by the data in table 1 . 


\section{Conclusions}

Dosimetry in small electron beams is challenging. The variation of the linac output is not easily predictable for the various combinations of applicator sizes and energies.

In this work high-energy small electron beams shaped by a commercial tubular applicator and field-defining end tubes were characterized by using a PTW Diode E dosimeter, together with a novel synthetic SCDD dosimeter, both initially validated in a standard $10 \times 10 \mathrm{~cm}^{2}$ field size by comparison with a plane-parallel ionization chamber. In particular, small electron beams with energies ranging from 6 to $15 \mathrm{MeV}$ and circular field sizes from $5 \mathrm{~cm}$ down to $2 \mathrm{~cm}$ diameter were investigated. PDD curves, beam profiles and OFs were measured by the two solid state detectors and the results were compared each other and found to be in good agreement within the stated uncertainties.

Two main effects were evidenced when using tubular applicators for the collimation of electron beams. Relative OFs larger than unity were found for field sizes of 5, 4 and $3 \mathrm{~cm}$ diameter at energies between 8 and $15 \mathrm{MeV}$. At the higher energies the linac output using small diameter tubular applicators increases to a large extent, up to $30 \%$ for $5 \mathrm{~cm}$ diameter field size at $15 \mathrm{MeV}$, in comparison with the output of the $10 \times 10 \mathrm{~cm}^{2}$ standard field size. Moreover, an increase of the OF values is observed for each diameter of the tubular applicator by increasing the beam energy while, at fixed energy, a decrease of OF can be noticed when reducing the field size.

Beam output in small electron beams formed by tubular applicators is influenced by electron scattering from applicator walls, air enclosed by applicator and scattering in the water. Dosimetric parameters in such fields, in particular OFs, differ a lot from those of fields shaped by typical electron applicators and inserts. It is recommended to measure dosimetric parameters for each field separately rather than attempt to interpolate values.

For dosimetry of small (a few cm diameter) electron beams plane-parallel ionization chambers have been reported to be not adequate due to their too large collecting electrode and consequent beam non-uniformity over the chamber area. In the present work both Si-D and SCDD dosimeters were found to be suitable to perform relative dosimetry, including the OFs determination, for electron beams shaped by tubular applicators with diameter from 5 to $2 \mathrm{~cm}$.

\section{Acknowledgments}

The authors wish to thank 'Fondazione Roma' for financial support and PTW-Freiburg for providing the encapsulation kit used for the diamond dosimeter investigated in the present paper and for helpful discussion. This work was also jointly funded by the EMRP participating countries within EURAMET and the European Union.

\section{References}

AAPM 1991 Clinical electron-beam dosimetry Report of AAPM Radiation Therapy Committee Task Group No. 25 Med. Phys. 1873

AAPM 2009 Recommendations for clinical electron beam dosimetry Supplement to the recommendations of Task Group 25 Report of AAPM Radiation Therapy Committee Task Group No. 70 Med. Phys. 363239

Almaviva $S$ et al 2010 Chemical vapor deposition diamond based multilayered radiation detector: physical analysis of detection properties J. Appl. Phys. 107014511

Almaviva S, Marinelli M, Milani E, Tucciarone A, Verona-Rinati G, Consorti R, Petrucci A, De Notaristefani F and Ciancaglioni I 2008 Synthetic single crystal diamond diodes for radiotherapy dosimetry Nucl. Instrum. Methods A 594 273-7 
Amin N, Heaton R, Norrlinger B and Islam M K 2011 Small field electron beam dosimetry using MOSFET detector J. Appl. Clin. Med. Phys. 12 50-7

Björk P, Knoos T and Nilsson P 2000 Comparative dosimetry of diode and diamond detectors in electron beams for intraoperative radiation therapy Med. Phys. 27 2580-8

Björk P, Knoos T and Nilsson P 2004 Measurements of output factors with different detector types and Monte Carlo calculations of stopping-power ratios for degraded electron beams Phys. Med. Biol. 49 4493-506

Ciancaglioni I, Marinelli M, Milani E, Prestopino G, Verona C, Verona-Rinati G, Consorti R, Petrucci A and De Notaristefani F 2012 Dosimetric characterization of a synthetic single crystal diamond detector in clinical radiation therapy small photon beams Med. Phys. 39 4493-501

Di Venanzio C, Marinelli M, Milani E, Prestopino G, Verona C, Verona-Rinati G, Falco M D, Bagalà P, Santoni R and Pimpinella M 2013 Characterization of a synthetic single crystal diamond Schottky diode for radiotherapy electron beam dosimetry Med. Phys. 40021712

Eveling J N, Morgan A M and Pitchford W G 1999 Commissioning a p-type silicon diode for use in clinical electron beams Med. Phys. 26 100-7

Griessbach I, Lapp M, Bohsung J, Gademann G and Harder D 2005 Dosimetric characteristics of a new unshielded silicon diode and its application in clinical photon and electron beams Med. Phys. 32 3750-4

Grusell E and Rikner G 1993 Linearity with dose rate of low resistivity p-type silicon semiconductor detectors Phys. Med. Biol. 38 785-92

Hogstrom K R and Almond P R 2006 Review of electron beam therapy physics Phys. Med. Biol. 51 R455-89

IAEA 2000 Absorbed dose determination in external beam radiotherapy: an international code of practice for dosimetry based on standards of absorbed dose to water Technical Report Series No. 398 (Vienna: IAEA)

ICRU 1984 Radiation dosimetry: electron beams with energies between 1 and $50 \mathrm{MeV}$ ICRU Report 35 (Bethesda, MD: International Commission on Radiation Units and Measurements)

Khan F M 2003 The Physics of Radiation Therapy 3rd edn (Philadelphia, PA: Lippincott Williams \& Wilkins)

Khan F M and Higgins P D 2001 Field equivalence for clinical electron beams Phys. Med. Biol. 46 N9-N14

Lax I and Brahme A 1980 Collimation of high energy electron beams Acta Radiol. Oncol. 19 199-207

Pimpinella M, Anton M, Rouijaa M and Stravato A 2012a Comparison of $D_{w}$ measurements by alanine and synthetic diamond dosimeters in photon beams with $1 \mathrm{~cm} \times 1 \mathrm{~cm}$ field size Metrologia 49 S211-4

Pimpinella M, Ciancaglioni I, Consorti R, Di Venanzio C, Guerra A S, Petrucci A, Stravato A and Verona-Rinati G 2012b A synthetic diamond detector as transfer dosimeter for $D_{w}$ measurements in photon beams with small field sizes Metrologia 49 S207-10

Pimpinella M, Mihailescu D, Guerra A S and Laitano R F 2007 Dosimetric characteristics of electron beams produced by a mobile accelerator for IORT Phys. Med. Biol. 52 6197-214

Rashid H, Islam M K, Gaballa H, Rosenow U F and Ting J Y 1990 Small-field electron dosimetry for the Philips SL25 linear accelerator Med. Phys. 17 710-4

Rikner G 1985 Characteristics of a p-Si detector in high energy electron fields Acta Radiol. Oncol. 24 71-4

Rogers D W O 2004 Accuracy of the burns equation for stopping-power ratio as a function of depth and $R_{50} M e d$. Phys. 31 2961-3

Saini A S and Zhu T C 2004 Dose rate and SDD dependence of commercially available diode detectors Med. Phys. 31 914-24

Song H, Ahmad M, Deng J, Chen Z, Yue N J and Nath R 2006 Limitations of silicon diodes for clinical electron dosimetry Radiat. Prot. Dosim. 120 56-9

Tapley N D 1976 Clinical Applications of the Electron Beam (New York: Wiley)

Thwaites D I, DuSautoy A R, Jordan T, McEwen M R, Nisbet A, Nahum A E and Pitchford W G 2003 IPEM (Institute of Physics and Engineering in Medicine) the IPEM code of practice for electron dosimetry for radiotherapy beams of initial energy from 4 to $25 \mathrm{MeV}$ based on an absorbed dose to water calibration Phys. Med. Biol. 48 2929-70

Vaeth J M and Meyer J L 1991 The Role of High Energy Electrons in the Treatment of Cancer (Frontiers of Radiation Therapy and Oncology vol 25) 25th Annu. San Francisco Cancer Symp. (San Francisco, CA: Karger)

Zhang G G, Rogers D W O, Cygler J E and Mackie T R 1999 Monte Carlo investigation of electron beam output factors versus size of square cutout Med. Phys. 26 743-50 